\title{
REGARDING THE PUTATIVE ECCENTRICITY OF CHARON'S ORBIT
}

\author{
S. Alan Stern, William F. Bottke, and Harold F. Levison \\ Department of Space Studies, Southwest Research Institute, Suite 400, 1050 Walnut Street, \\ Boulder, CO 80302; astern@swri.edu \\ Received 2002 August 13; accepted 2002 October 31
}

\begin{abstract}
Based on astrometry from an orbit derived by Hubble Space Telescope imagery, Charon's orbital eccentricity has been reported to be in the range of $0.003-0.008$. Solar and planetary tides are orders of magnitude too small to induce the reported eccentricity. This nonzero value, if correct, therefore indicates some significant forcing against the two-body tidal equilibrium value, which should formally be zero. Here we follow up on a preliminary study to investigate whether the reported eccentricity of Charon's orbit could be due to gravitational perturbations by Kuiper belt object (KBO) flybys through the Pluto-Charon system and KBO impacts directly onto Pluto and Charon. We find it is unlikely that Charon's reported eccentricity could be caused by this effect. Although we cannot rule out some additional source of eccentricity excitation (e.g., an undiscovered satellite in the system, or a Kozai resonance), our analysis indicates it is plausible that Charon's actual orbital eccentricity is substantially smaller than the 0.003 lower limit reported previously.
\end{abstract}

Key words: Kuiper belt — planets and satellites: individual (Charon)

\section{INTRODUCTION}

Some years ago, Hubble Space Telescope-derived astrometry of Charon's orbit about Pluto provided evidence for a significant, nonzero orbital eccentricity of Charon, with likely values in the range $0.003-0.008$ (Tholen \& Buie 1997). This report was somewhat of a surprise to many, and was met by some with skepticism, in that it had been expected that tidal evolution in the Pluto-Charon system would drive Charon's equilibrium eccentricity to values negligibly close to zero (see Dobrovolskis, Peale, \& Harris 1997). The validity of the reported eccentricity was considered further suspect by Tholen \& Buie's (1997) finding of a line of apsides pointed toward Earth.

The reported eccentricity of Charon's orbit cannot be the result of the incomplete tidal spin-down of the PlutoCharon binary, because this timescale is very short $\left(\sim 10^{7}\right.$ yr) compared with the age $(4.6 \mathrm{Gyr})$ of the solar system (e.g., Peale 1986). Charon's reported eccentricity also cannot be attributed to solar perturbations on Charon's orbit, which can only induce an eccentricity of $\sim 5 \times 10^{-9}$ (Weissman, Dobrovolskis, \& Stern 1989). In what follows, we investigate the possibility that either physical collisions on, or flyby perturbations of, Pluto-Charon could induce the reported eccentricity, as first posited in a brief study (Levison \& Stern 1995).

\section{COMPUTATIONAL TECHNIQUE}

To test this idea, we constructed a Monte Carlo code that sends model Kuiper belt object (KBO) populations through the Pluto-Charon system, in order to derive the likelihood that the reported system eccentricity is due to perturbations or impacts by KBOs.

Toward this end, we calculated the intrinsic collision probability $P_{i}$ (i.e., the number of impacts per unit surface area per unit time) of KBOs with Pluto. We did this using the orbits of all classical and resonant KBOs with multipleopposition orbits and semimajor axis $a<50 \mathrm{AU}$; we note that $P_{i}$ includes the effect of gravitational focusing by Pluto. Scattered-belt KBOs with large semimajor axes were not included; owing to their higher encounter speeds and lower number densities in the 30-50 AU zone where Pluto-Charon resides, their effects are much smaller.

To account for first-order detection biases in the Kuiper belt population, we debiased the population by weighting the data points by the factor $\beta=\sin i$, where $i$ is the orbital inclination (see, e.g., Brown 2001).

We integrated the orbits of the debiased KBO population for $1 \mathrm{Gyr}$, tracking the rate at which they penetrated PlutoCharon's Hill sphere. This integration included the Sun, the four giant planets, Pluto, and test particles representing each KBO. From this calculation we derived a mean $P_{i}=4.2 \times 10^{-22} \mathrm{~km}^{-2} \mathrm{yr}^{-1}$, in accord with an estimate one can easily derive from a simple particle-in-a-box model. The mean encounter velocity of KBOs with respect to PlutoCharon's center of mass at their Hill sphere crossing was found to be $2.1 \mathrm{~km} \mathrm{~s}^{-1}$, the median encounter velocity was $2.0 \mathrm{~km} \mathrm{~s}^{-1}$, and the bias-corrected rms velocity of KBOs relative to Pluto is $2.3 \mathrm{~km} \mathrm{~s}^{-1}$.

Using the estimated intrinsic collision probability, we constructed a Monte Carlo code to track the effects of KBO close encounters and collisions on Pluto and Charon. We did this in order to determine the effect such encounters and collisions induce on Charon's orbital eccentricity $e$. We assumed an initial Charon orbit of $a_{\mathrm{C}}=19,600 \mathrm{~km}$, with $e_{\mathrm{C}}=0$ and $i_{\mathrm{C}}=0$ (see, e.g., Tholen \& Buie 1997). We assumed Pluto and Charon radii of 1180 and $600 \mathrm{~km}$, respectively; we assumed Pluto and Charon masses of $1.38 \times 10^{25}$ and $1.86 \times 10^{24} \mathrm{~g}$, respectively, corresponding to Pluto and Charon densities of $2 \mathrm{~g} \mathrm{~cm}^{-3}$.

To generate various KBO impactor populations, we assumed a power-law size-frequency distribution of KBOs with differential power-law indices $q$ of $-4.5,-4.0$, and -3.5 , which bound the likely population structure of the Kuiper belt (e.g., Jewitt \& Luu 2000). The number of KBOs with $D>100 \mathrm{~km}$ and $D<660 \mathrm{~km}$ was set to $5 \times 10^{4}$ and then $1.5 \times 10^{5}$ in successive runs for each power law. These power-law size distributions were extended down to $100 \mathrm{~m}$ for collisions, and $5 \mathrm{~km}$ for close encounters. (A smaller size cutoff for the close encounters was not possible, owing to 
computational limitations in the number of flybys that would have to be treated. Order-of-magnitude estimates show that even $5 \mathrm{~km}$ bodies cannot individually perturb Charon's orbital eccentricity near the $10^{-5}$ level, far below the 0.003 value reported in the literature.)

We used random deviates to select the $\mathrm{KBO}$ diameter and a distance of closest approach $b$ of each KBO in the Monte Carlo runs. We computed KBO masses assuming spherical shapes and a bulk density of $2 \mathrm{~g} \mathrm{~cm}^{-3}$. For simplicity, we assumed the mass ratio of Pluto to Charon is simply the volume ratio of the two bodies.

We computed the mean time between encounters separately, as a function of $\mathrm{KBO}$ size, for collisions and encounters using $T_{\mathrm{enc}}=\left(P_{i} N_{\mathrm{KBO}} b^{2}\right)^{-1}$, where $N_{\mathrm{KBO}}$ is the number of KBOs in a given size bin and $b$ is the largest separation distance capable of producing an effect of interest on PlutoCharon; we accepted $b$ up to twice the semimajor axis of Charon's orbit. We computed the time between physical collisions the same way, but with $b$ set to the radius of the target body (Pluto or Charon). The actual interval between each successive collision or close encounter was then modeled as a Poisson process around this mean time.

If no physical collision occurred, then, using the impulse approximation and a random orientation of the encounter relative to the binary, we calculated a velocity change for Charon relative to Pluto, from which we in turn computed the change in orbital elements of the binary. If a physical collision occurred, we followed the same procedure, but we employed Zahnle, Dones, \& Levison's (1998) collision probability function to determine the location of the collision relative to the apex of orbital motion (see their eqs. [31]-[32]). We also assumed wholly inelastic collisions.

We modeled the eccentricity of Charon as declining between excitation events as the result of a relaxation toward tidal equilibrium. In other words, we assume that Charon is always evolving back to the synchronous position after a perturbation. To do this, we followed the (constant time lag) formalism developed by Goldreich \& Soter (1966; see also Kaula 1964; Touma \& Wisdom 1994), as Weissman et al. (1989) did. For lack of a better value to use, we arbitrarily set the phase lag for the bulge raised on Pluto by Charon to be 20 minutes. In terms of Pluto's $Q$-value, this becomes $Q_{\mathrm{P}}=\csc 2 \Lambda$, where the lag angle $\Lambda=(20$ minutes $)(\Omega-n)$ with $n$ being Charon's mean motion and $\Omega$ being the spin rate of Pluto. We include the $\Omega$ and mean motion terms in $Q_{\text {P }}$ so we can correctly solve the differential equations that govern how Charon's eccentricity evolves with time. The quantity $Q_{\mathrm{C}}$ was set to 100 (e.g., Knopoff 1964).

We assumed a rigidity $\mu$ for Pluto of that of water ice $\left(4 \times 10^{10} \mathrm{dyn}^{\mathrm{cm}}{ }^{-2}\right)$. For Charon, we assumed what we believe are bounding cases of rigidity, that is, either a weak case, that of water ice $\left(4 \times 10^{10} \mathrm{dyn} \mathrm{cm}^{-2}\right.$; Proctor 1966), or a strong case - a rocklike rigidity like that of the Moon $\left(6.5 \times 10^{11} \mathrm{dyn} \mathrm{cm}^{-2}\right.$; Nakamura, Latham, \& Dorman 1976); these correspond to tidal damping timescales of 17 and $202 \mathrm{Myr}$, respectively.

\section{MODEL RUNS AND RESULTS}

To test the role that $\mathrm{KBO}$ close encounters and collisions may play in exciting Charon's orbital eccentricity away from its zero (equilibrium tidal) value, we made a series of runs of the model described above. In total, 12 separate model runs were conducted. As shown in Table 1, these consisted of two sets of six runs in which we varied the size distribution and total population of KBOs. As noted above, each set of six runs used three combinations of KBO differential population power-law indices $(q=-3.5,-4.0,-4.5)$ and two combinations of $\mathrm{KBO}$ populations $[N(D>100$ $\mathrm{km})=5 \times 10^{4}$ and $N(D>100 \mathrm{~km})=1.5 \times 10^{5}$ ] between 30 and $50 \mathrm{AU}$. These six KBO population variants span the plausible range of KBO populations (e.g., Jewitt \& Luu 2000; Trujillo, Jewitt, \& Luu 2001; Gladman et al. 2001); these six population-spanning runs were repeated for each of the two solid-body rigidities of Charon that we used, as also discussed above.

Figure 1 presents the results of a sample model run (case $4 \mathrm{~S}$ ), showing Charon's eccentricity evolution as a function of time; note the rarity with which its orbital eccentricity reaches or exceeds the nominal reported value of 0.003 . Figure 2 presents all 12 model runs, showing the integrated fraction of time eccentricity reaches or exceeds any given eccentricity. Median eccentricities for Charon's orbit were found to range from just below $10^{-5}$ to just below $10^{-3}$. Table 2 provides the fraction of time during which $e>0.003$ for each of the 12 model cases.

As stated previously, the relative frequency of $\mathrm{KBO}$ close encounters with the Pluto-Charon system can be found

TABLE 1

Run CASe Descriptions

\begin{tabular}{|c|c|c|c|c|}
\hline $\begin{array}{l}\text { Run } \\
\text { Case }\end{array}$ & $\begin{array}{l}\text { Assumed Number of } \\
\text { KBOs }(D>100 \mathrm{~km})\end{array}$ & $\begin{array}{l}\text { Power-Law } \\
\text { Index } q\end{array}$ & $\begin{array}{l}\text { Resulting 30-50 AU } \\
\text { Kuiper Belt Mass }\end{array}$ & $\begin{array}{l}\text { Charon's Bulk } \\
\text { Rigidity }\end{array}$ \\
\hline $1 \mathrm{~W} \ldots \ldots \ldots \ldots \ldots$ & $5 \times 10^{4}$ & -3.5 & 0.11 & Water ice \\
\hline $2 \mathrm{~W} \ldots \ldots \ldots \ldots \ldots$ & $5 \times 10^{4}$ & -4.0 & 0.24 & Water ice \\
\hline $3 \mathrm{~W} \ldots \ldots \ldots \ldots \ldots$ & $5 \times 10^{4}$ & -4.5 & 2.10 & Water ice \\
\hline $4 \mathrm{~W} \ldots \ldots \ldots \ldots \ldots$ & $1.5 \times 10^{5}$ & -3.5 & 0.33 & Water ice \\
\hline $5 \mathrm{~W} \ldots \ldots \ldots \ldots \ldots$ & $1.5 \times 10^{5}$ & -4.0 & 0.71 & Water ice \\
\hline 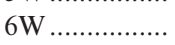 & $1.5 \times 10^{5}$ & -4.5 & 6.20 & Water ice \\
\hline 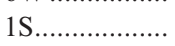 & $5 \times 10^{4}$ & -3.5 & 0.11 & Bulk lunar \\
\hline $2 \mathrm{~S} \ldots \ldots \ldots \ldots \ldots \ldots$ & $5 \times 10^{4}$ & -4.0 & 0.24 & Bulk lunar \\
\hline 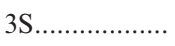 & $5 \times 10^{4}$ & -4.5 & 2.10 & Bulk lunar \\
\hline $4 \mathrm{~S} \ldots \ldots \ldots \ldots \ldots \ldots$ & $1.5 \times 10^{5}$ & -3.5 & 0.33 & Bulk lunar \\
\hline $5 \mathrm{~S} \ldots \ldots \ldots \ldots \ldots \ldots$ & $1.5 \times 10^{5}$ & -4.0 & 0.71 & Bulk lunar \\
\hline $6 \mathrm{~S} \ldots \ldots \ldots \ldots \ldots \ldots$ & $1.5 \times 10^{5}$ & -4.5 & 6.20 & Bulk lunar \\
\hline
\end{tabular}

a For KBO diameters 0.1-660 km, in Earth masses. 


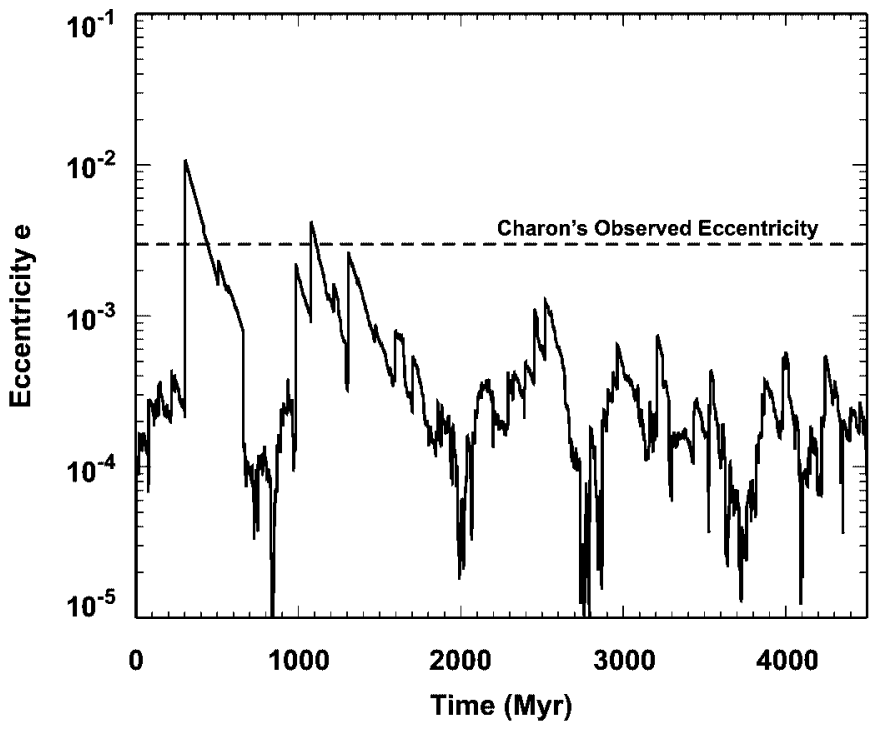

FIg. 1.- Results of a sample model run (case 4S; see Table 1), showing Charon's eccentricity evolution as a function of time; note the rarity with which its orbital eccentricity reaches or exceeds the reported minimum value of 0.003 .
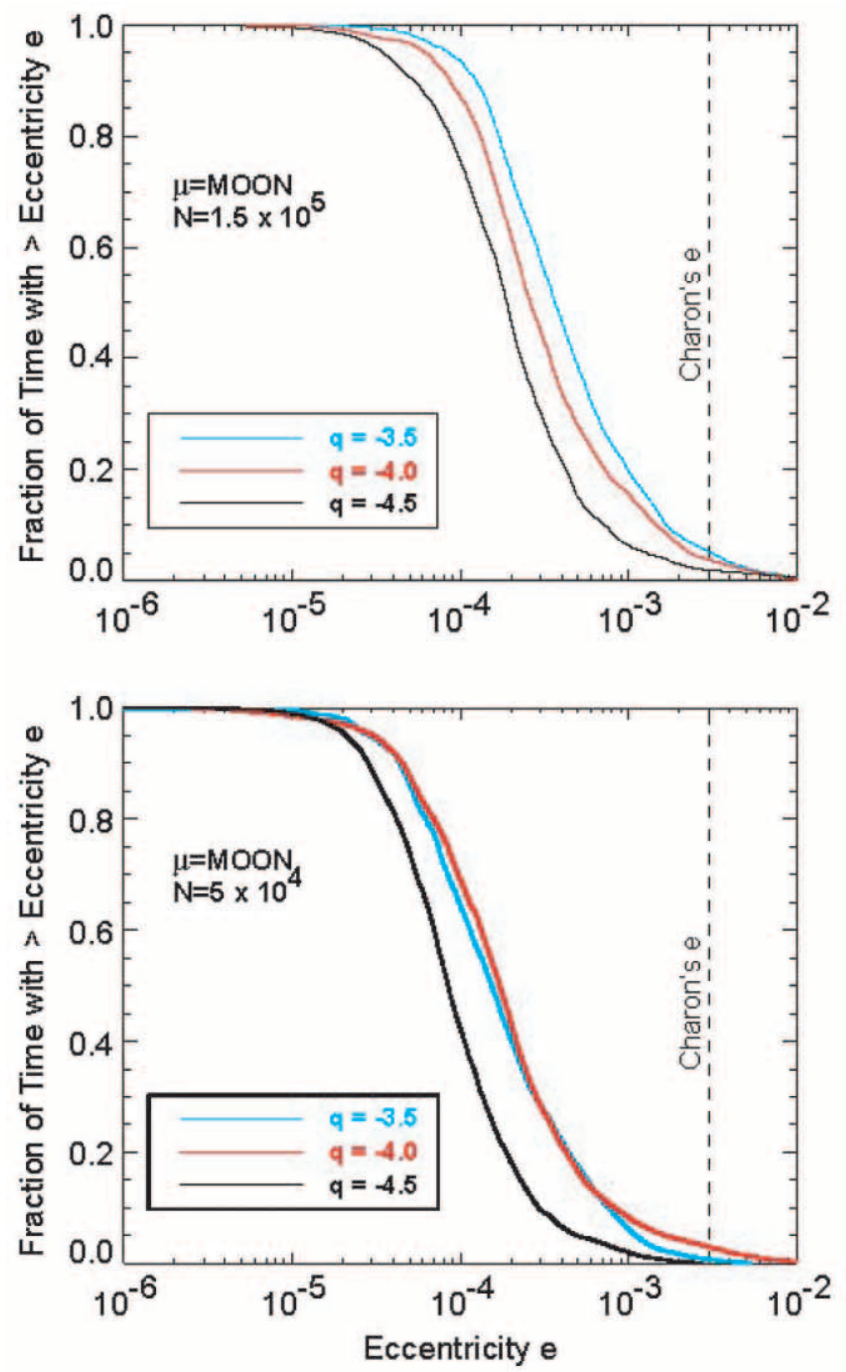

TABLE 2

Fraction of Time Charon's Eccentricity Was Found TO EXCEED 0.003

\begin{tabular}{|c|c|c|c|}
\hline $\begin{array}{l}\text { Run } \\
\text { Case }\end{array}$ & $\begin{array}{l}\text { Assumed Number of } \\
\operatorname{KBOs}(D>100 \mathrm{~km})\end{array}$ & $\begin{array}{l}\text { Power-Law } \\
\quad \text { Index } q\end{array}$ & $\begin{array}{c}\text { Temporal Fraction } \\
e>0.003\end{array}$ \\
\hline $1 \mathrm{~W} \ldots \ldots . .$. & $5 \times 10^{4}$ & -3.5 & 0.003 \\
\hline $2 \mathrm{~W} \ldots \ldots \ldots$ & $5 \times 10^{4}$ & -4.0 & 0.002 \\
\hline $3 \mathrm{~W} . . . \ldots . .$. & $5 \times 10^{4}$ & -4.5 & 0.001 \\
\hline $4 \mathrm{~W} \ldots \ldots . .$. & $1.5 \times 10^{5}$ & -3.5 & 0.003 \\
\hline $5 \mathrm{~W} \ldots \ldots \ldots$ & $1.5 \times 10^{5}$ & -4.0 & 0.001 \\
\hline $6 \mathrm{~W} . . . \ldots \ldots . .$. & $1.5 \times 10^{5}$ & -4.5 & 0.001 \\
\hline 1S.............. & $5 \times 10^{4}$ & -3.5 & 0.008 \\
\hline $2 \mathrm{~S} \ldots \ldots \ldots \ldots$ & $5 \times 10^{4}$ & -4.0 & 0.003 \\
\hline $3 \mathrm{~S} \ldots \ldots \ldots . .$. & $5 \times 10^{4}$ & -4.5 & $<0.001$ \\
\hline $4 \mathrm{~S} \ldots \ldots \ldots$ & $1.5 \times 10^{5}$ & -3.5 & 0.052 \\
\hline $5 \mathrm{~S} \ldots \ldots \ldots . .$. & $1.5 \times 10^{5}$ & -4.0 & 0.037 \\
\hline $6 \mathrm{~S} \ldots \ldots \ldots . . .$. & $1.5 \times 10^{5}$ & -4.5 & 0.019 \\
\hline
\end{tabular}

using the equation $T_{\text {enc }}=\left(P_{i} N_{\mathrm{KBO}} b^{2}\right)^{-1}$, while the collision frequency can be found by replacing $b^{2}$ with the squared radii of Pluto and Charon. We found that for a given KBO, close encounters through the Pluto-Charon system at a distance of $b=a_{\mathrm{C}}$ occur just over 200 times more frequently
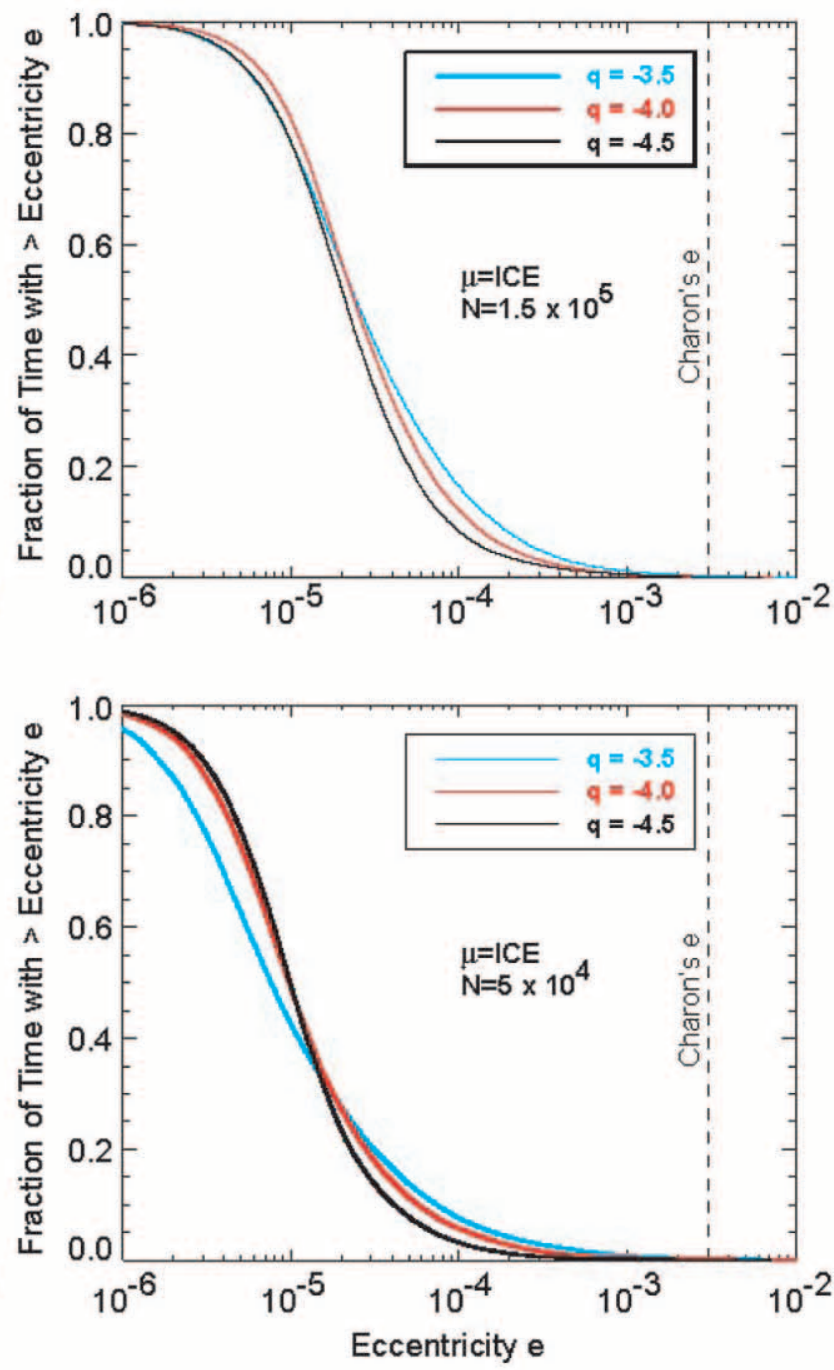

FIG. 2.-All 12 model runs, showing the integrated fraction of time that eccentricity reaches or exceeds any given eccentricity. The vertical lines show the fraction of time Charon spent with $e=0.003$ in all 12 of these run cases; the same is also given in Table 1. 
than collisions. Collisions, on the other hand, deliver more momentum change per unit mass than close encounters, such that smaller (and presumably more numerous) KBOs may also be important in changing Charon's eccentricity. The importance of close encounters relative to that of collisions is difficult to specify uniquely, because factors such as the KBO size-frequency distribution, the interloper-PlutoCharon geometry at encounter or collision, the encounter velocity, and the stochastic nature of encounters and collisions all play important roles. However, from test runs during which we turned off physical collisions but kept close encounters, we found that collisions typically generate a median Charon orbital eccentricity that is typically $5-10$ times smaller than that caused by close encounters.

To raise the temporal fraction in which $e>0.003$ to a high probability (e.g., 25\%) would require that the product of $Q$ and rigidity $\mu$ be higher by 1 or more orders of magnitude than our highest value $\left(Q=100, \mu=6.5 \times 10^{11}\right.$ dyn $\mathrm{cm}^{-2}$ [i.e., rock]). While not impossible, we consider this implausible based on the wide range of solid solar system bodies for which $Q$ and $\mu$ are known.

Our results yield far smaller mean random eccentricities for Charon, and far smaller time fractions when Charon's eccentricity exceeds 0.003 , than those of Levison \& Stern (1995). This is because of our refined understanding of the population structure of the Kuiper belt, which contains fewer larger bodies than was expected in 1995.

In conclusion, we find that the $\mathrm{KBO}$ excitation mechanism fails to produce Charon orbital eccentricities as high as those reported by Tholen \& Buie (1997) except rarely and, even then, only for certain Kuiper belt models. In general, cases with greater large $\mathrm{KBO}$ populations and more icelike bulk rigidities maximize the fraction of time Charon has higher orbital eccentricity.

\section{WHAT THEN OF CHARON'S REPORTED ECCENTRICITY?}

We have found that the likelihood of KBO collisions or close encounters exciting Charon's orbital eccentricity to the value reported from Hubble Space Telescope observations, $e=0.003$, is small, less than $5 \%$ for the most optimistic case we ran and less than $0.3 \%$ for most other model run cases.

As noted at the start of this paper, solar and planetary tides have also been shown to be incapable of generating a Charon eccentricity close to 0.003 . Could it be that Charon's orbital eccentricity is excited by some other mechanism? Perhaps. One possibility that comes to mind would be the presence of an undetected satellite in the system. Stern et al. (1994) provides the most stringent applicable constraints on additional satellites that might lie in the Pluto-Charon system. That paper reported that it is plausible that satellites with radii of up to $140 \mathrm{~km}$ could remain undetected between Pluto's Roche limit, near $0.15 a_{\mathrm{C}}$, and $\sim 0.5 a_{\mathrm{C}}$. Such satellites are large enough to induce orbital eccentricities of order 0.003 on Charon. Outside of Charon's orbit, stable orbits were found beginning at $\sim 2 a_{\mathrm{C}}$, where objects of $42 \mathrm{~km}$ radius or smaller may be lurking. However, we discount the possibility that an undiscovered satellite of sufficient size to generate $e>0.003$ remains undiscovered in the Pluto system, owing to (1) the limited parameter space in which sufficiently massive satellites could orbit and yet remain undetected and (2) the fact that any satellite interior to Charon would likely have to have been emplaced after Charon's suspected outward orbital migration under tides (see, e.g., Peale 1986).

The key to resolving the discrepancy between our results and the reported eccentricity of Charon's orbit will depend on new observations of Charon's orbit, searches for additional satellites, and clever thinking by theorists about possible resonances that might excite the orbital eccentricity. We do note, however, that it remains to be studied whether a Kozai mechanism, eccentricity resonances (e.g., perhaps related to the inclination resonance effects discussed by Rubincam [2000]), or a strong libration of Pluto coupled to a significant Plutonian $J_{2}$ could drive a sufficiently large equilibrium eccentricity.

Nevertheless, until such time as one of those routes points definitively to a resolution of the discrepancy that we have discovered, we believe it is advisable that workers consider Charon eccentricities in excess of $10^{-3}$, or even $10^{-4}$, with a degree of skepticism.

This work was supported by the NASA Origins and Planetary Geology and Geophysics Programs. We thank David Tholen and David Rubincam for helpful discussions. Luke Dones, Alan Harris, Alessandro Morbidelli, Stan Peale, and Bill Ward each provided valuable comments on this paper. Tony Dobrovolskis provided helpful comments as the paper's referee.

\section{REFERENCES}

Brown, M. E. 2001, AJ, 121, 2804

Dobrovolskis, A. R., Peale, S. J., \& Harris, A. W. 1997, in Pluto and Charon, ed. S. A. Stern \& D. J. Tholen (Tucson: Univ. Arizona Press), 159

Gladman, B., Kavelaars, J. J., Petit, J.-M., Morbidelli, A., Holman, M. J., \& Loredo, T. 2001, AJ, 122, 1051

Goldreich, P., \& Soter, S. 1966, Icarus, 5, 375

Jewitt, D. C., \& Luu, J. X. 2000, in Protostars and Planets IV, ed. V. Mannings, A. P. Boss, \& S. S. Russell (Tucson: Univ. Arizona Press), 1201

Kaula, W. M. 1964, Rev. Geophys., 2, 661

Knopoff, L. 1964, Rev. Geophys., 2, 625

Levison, H. F., \& Stern, S. A. 1995, in Lunar and Planetary Science XXVI (Houston: Lunar Planet. Inst.), 841
Nakamura, Y., Latham, G. V., \& Dorman, H. J. 1976, in Lunar Science VII (Houston: Lunar Planet. Inst.), 602

Peale, S. J. 1986, in Satellites, ed. J. A. Burns \& M. S. Matthews (Tucson: Univ. Arizona Press), 159

Proctor, T. M. 1966, J. Acoust. Soc. Am., 39, 972

Rubincam, D. P. 2000, J. Geophys. Res., 105, 26745

Stern, S. A., Parker, J. W., Duncan, M. J., Snowdall, J. C., Jr., \& Levison, H. F. 1994, Icarus, 108, 234

Tholen, D. J. \& Buie, M. W. 1997, Icarus, 125, 245

Touma, J., \& Wisdom, J. 1994, AJ, 108, 1943

Trujillo, C. A., Jewitt, D. C., \& Luu, J. X. 2001, AJ, 122, 457

Weissman, P. R., Dobrovolskis, A. R., \& Stern, S. A. 1989, Geophys. Res. Lett., 16, 1241

Zahnle, K., Dones, L., \& Levison, H. F. 1998, Icarus, 136, 202 